\title{
Executive Function Performance in Young Adults When Cycling at an Active Workstation: An fNIRS Study
}

\author{
Tao Huang ${ }^{1}$, Qian Gu ${ }^{1}$, Zhangyan Deng ${ }^{1,2}$, Chilun Tsai ${ }^{1}$, Yue Xue ${ }^{1}$, Jimeng Zhang ${ }^{1}$, \\ Liye Zou ${ }^{3}{ }^{(1)}$, Zuosong Chen ${ }^{1}$ and Kun Wang ${ }^{1, *(1)}$ \\ 1 Department of Physical Education, Shanghai Jiao Tong University, Shanghai 200240, China; \\ taohuang@sjtu.edu.cn (T.H.); sjtuguqian@sjtu.edu.cn (Q.G.); zhangyandeng@sjtu.edu.cn (Z.D.); \\ henry41202@yahoo.com.tw (C.T.); xy0927@sjtu.edu.cn (Y.X.); simon2017@sjtu.edu.cn (J.Z.); \\ zschen1971@126.com (Z.C.) \\ 2 Graduate school of Education, Shanghai Jiao Tong University, Shanghai 200240, China \\ 3 Lifestyle (Mind-Body Movement) Research Center, College of Sports Science, Shenzhen University, \\ Shenzhen 518060, China; liyezou123@gmail.com \\ * Correspondence: wangkunz@sjtu.edu.cn; Tel.: +86-021-54743157
}

Received: 13 February 2019; Accepted: 24 March 2019; Published: 28 March 2019

\begin{abstract}
Background: This study aimed to investigate the effects of self-paced cycling at an active workstation on executive functions and cortical activity. Methods: In a crossover study design, 37 young adults ( $45.9 \%$ females) were randomly assigned to the following two task conditions: (1) performing cognitive tests during sitting, (2) performing cognitive tests while cycling at an active workstation. Executive functions were assessed by the Stroop color and word test and the task-switching paradigm. Cortical activity was monitored using a multi-channel functional near-infrared spectroscopy (fNIRS) system. Results: The behavioral results showed that there were no significant differences on the Stroop interference effects $(P=0.66)$ between the sitting and the cycling conditions. In all probability, no differences on the global switch costs $(P=0.90)$ and local switch costs $(P=0.67)$ were observed between the sitting and the cycling conditions. For the fNIRS results, the oxygenated hemoglobin (oxy- $\mathrm{Hb})$ in response to the Stroop interference in channels 5, 10, and 12 were decreased during the cycling condition (all Ps $<0.05$, FDR-corrected). Conversely, the oxy- $\mathrm{Hb}$ associated with the global switch costs in channels 3, 29, and 31 were increased during the cycling condition (all Ps $<0.05$, FDR-corrected). Conclusions: The findings indicated that behavioral performances on executive functions were not affected by cycling at an active workstation, while cognitive resources were reallocated during cycling at an active workstation.
\end{abstract}

Keywords: sedentary behavior; executive functions; active workstation; exercise; fNIRS

\section{Introduction}

Sedentary behavior refers to any activities when people are awake, where their energy expenditure is less than 1.5 MET (metabolic equivalent) [1]. It is widely accepted that sedentary behaviors and physical inactivity have adverse impacts on an individual's health, but also bring about substantial medical and economic burden [2,3]. Such results have drawn increasing public attention in recent years. Prolonged sedentary time is independently associated with increased risk of developing noncommunicable diseases, such as obesity [4], diabetes [5], cardiovascular diseases [6], and mental disorders $[7,8]$. Conversely, breaking up sedentary time has been shown to contribute immensely to physical and mental health, such as favorable metabolic changes $[9,10]$. 
Young adults usually spend a great amount of sitting time at work or study in addition to other forms of sedentary behaviors. Therefore, it is especially urgent to intervene sedentary behaviors that are essential for chronic disease prevention in this age group. The recent evidence suggests that implementation of active workstations may be a promising intervention strategy for breaking up sedentary time at the workplace [11-14]. For example, active workstations provided the opportunity to perform low-intensity exercise or to stand while working or studying, which led to reduced sedentary time $[15,16]$. Furthermore, it consistently demonstrated that active workstations improved anthropometric indicators (e.g., body weight [17,18] and waist circumstances [19]), cardiovascular metabolic risk indicators (e.g., cholesterol [19], high-density lipoprotein [20], and postprandial blood glucose [21]).

People have debated whether study or work at an active workstation influences cognitive ability (e.g., executive functions) and further interferes with working performance and efficiency [22]. Executive functions are a set of higher-order cognitive processes that are needed by people to perform the activities of daily living. They mainly consist of inhibitory control, working memory, cognitive flexibility, planning, and reasoning [23]. Furthermore, executive functions play a critical role in academic and professional success [23], socio-emotional development [24], and physical and mental health [25]. Some studies demonstrated that working at an active workstation did not adversely affect executive function performance [26-30], whereas others demonstrated a positive effect using active workstations [31], or they observed a selective impairment on a subdomain of executive functions [32]. It is worth noting that previous studies mainly focused on the end-state effects of using active workstations on behavioral performance of executive functions, rather than the in-task neural cognitive processes [28]. Functional near-infrared spectroscopy (fNIRS), a non-invasive technique to monitor the changes of concentration of oxygenated (oxy- $\mathrm{Hb}$ ) and deoxygenated (deoxy- $\mathrm{Hb}$ ) hemoglobin in the blood, is commonly used to assess cerebral activity. Recent evidence suggests that fNIRS is suitable for measuring the real-time changes in brain activity during exercise [33].

Therefore, to clarify the effects of using active workstations on executive function performance and brain activity, the fNIRS approach was used to measure the cortical activity during executive function tasks, performed by Chinese university students while cycling at an active workstation.

\section{Materials and Methods}

\subsection{Participants}

Forty-one right-handed participants were recruited from two universities in Shanghai, China. All participants were undergraduate students or master's students. Among the participants, one was excluded due to abnormal color vision and three participants did not complete the study protocol. Finally, 37 participants who completed the full protocol were included for data analysis. Written informed consents were obtained from all participants. The study protocol was approved by the ethical committee at Shanghai Jiao Tong University (ethical code: 20170100). The study was conducted in accordance with the ethical requirement of the Declaration of Helsinki.

\subsection{Procedures}

The study adopted a randomized crossover design. The experimental design and protocol are shown in Figure 1. Participants reported to the laboratory on two separate occasions, and each occasion was separated by at least 48 hours. At one experimental session, the participants performed the sitting condition, in which they completed two executive function tasks (i.e., Stroop color and word test, and task-switching). In this condition, the participants sat at an active cycling workstation (Loctek V9, Loctek Ergonomic Technology Corp., Ningbo, China) without performing any pedaling. At the other experimental session, the participants performed the cycling condition, and they completed the same cognitive task while cycling. A specific workload was not assigned, and therefore participants were allowed to cycle at a speed that was comfortable to them. The Borg rating of perceived exertion (RPE) 
scale was used to measure perceived exertion before and after the experimental sessions. The heart rate (HR) of participants was monitored throughout the two experimental sessions using a HR monitor (Suunto M5, SUUNTO Inc., Vantaa, Finland). In order to avoid potential order effects, participants performed the two experimental session in a counterbalanced order.

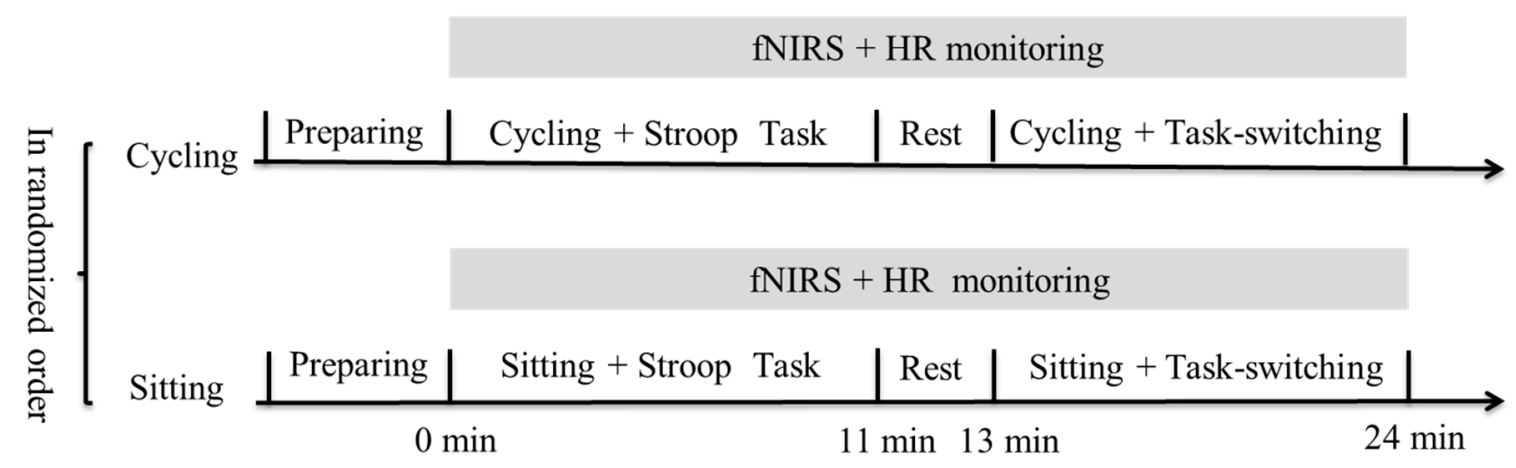

Figure 1. Chart of experimental design and protocol.

\subsection{Measurements of Executive Functions}

The participants' performance on executive functions were measured by a computerized Stroop color and word test, and a task-switching paradigm. The two tasks were programmed using E-Prime 2.0 (Psychology Software Tools, Inc., Sharpsburg, PA, USA) in an event-related design.

\subsubsection{Stroop Color and Word Test}

The computerized Stroop task was programmed according to previous studies based on fNIRS [34]. For each trial, two rows of stimuli were displayed at the center of the screen. The bottom row displayed a Chinese word (i.e., red, green, blue, or yellow) printed in white, and the upper row displayed a Chinese word (neutral word or color word) printed in four colors (red, green, blue, or yellow). Two experimental conditions (neutral and incongruent) were defined in the present study based on the feature of upper stimuli. For the neutral condition, the upper row was a neutral word and the meaning of the word was not related to the color. For the incongruent condition, the upper row was a color word, and the color of the word did not denote the meaning of the word (e.g., the word "yellow" printed in red). Participants were instructed to decide whether the color of the upper row corresponded to the meaning of the bottom row. The Stroop test consisted of 48 judgment trials (24 neutral trials and 24 incongruent trials). For each trial, the fixation presentation varied randomly between 400 and $600 \mathrm{~ms}$. Each stimulus was displayed on the screen for $400 \mathrm{~ms}$, and then the response screen was displayed for $1600 \mathrm{~ms}$, with an interval blank screen displayed for 11,000 ms. The order of the trials was randomly presented.

In addition to the accuracy and the reaction time (RT), the Stroop interference effects were derived by the contrast of RT between the incongruent condition and the neutral condition [35].

\subsubsection{Task-Switching}

The computerized task-switching paradigm adopted in this study was based on a previous study [36]. On each trial, either a red or green number (1-9, exclude 5) was displayed at the center of the screen. For the red number trials, participants were asked to press " $X$ " on the keyboard if the number was smaller than 5 and to press the key " $\mathrm{M}$ " if the number was larger than 5 . For the green number trials, participants were asked to press the key " $X$ " if an odd number was presented and to press the key " $\mathrm{M}$ " if an even number was presented. The formal test began with the pure task (20 trials) in which the "large or small" and "odd or even" trials were respectively displayed. The participants were to maintain their response pattern in a task sequence. Following the pure task, the mixed task (32 trials) was displayed where the "large or small" and "odd or even" trials alternated after every 
second trial (in ABBA sequence). Therefore, the participants switched their response pattern in 16 trials, and they maintained their response pattern in the other trials. The changes in response pattern created a behavioral or cognitive cost between the non-switch condition and the switch condition, which is referred to as the global (mixed task vs. pure task) or local (switch trials in mixed task vs. non-switch trial in the mixed task) switch costs $[37,38]$. For each trial, the fixation presented varied randomly between 400 and $600 \mathrm{~ms}$. The stimulus was displayed on the screen for $200 \mathrm{~ms}$, and then the response screen was displayed for $1800 \mathrm{~ms}$, with an interval blank screen was displayed for 10,000 ms. The stimuli's digit was randomly presented.

\section{4. fNIRS Measurements and Data Preprocessing}

The multi-channel continuous wave fNIRS system NIRScout (NIRX Medical Technologies LLC, New York, NY, USA, Sampling rate $=3.91 \mathrm{~Hz}$ ) was used to monitor hemodynamic responses. Sixteen dual-wavelength source probes $(760$ and $850 \mathrm{~nm}$ ) and 12 optical detector probes covered several 10-10 electroencephalography (EEG) positions. A nearby source-detector was paired to obtain 38 channels, and the distance between them was $3 \mathrm{~cm}$. On the basis of the probabilistic estimation method $[39,40]$, the projection of each channel in the brain region was estimated (Figure 2). The MNI data of all coordinates and the image reconstruction software package was obtained from Jichi medical university (http:/ / www.jichi.ac.jp/brainlab/tools.html).

A

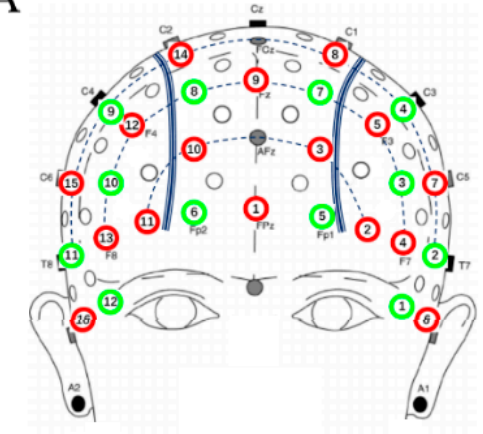

B

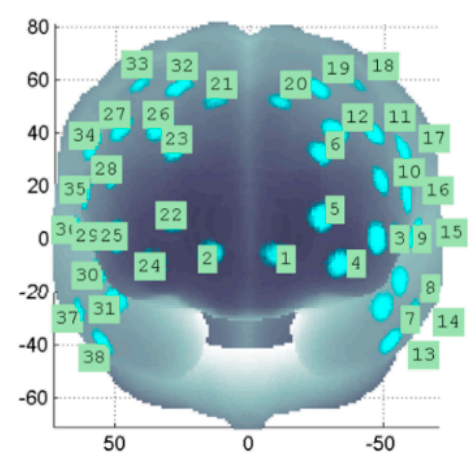

Figure 2. Location of the functional near-infrared spectroscopy (fNIRS) probe. (A) Spatial profiles of optical sources (red circles) and detector probes (green circles); (B) Estimated position of each channel.

The Matlab-based open-source software package HOMER2 v. 2.3 (https:/ /homer-fnirs.org/) was used to process the fNIRS data [41]. The modified Beer-Lambert Law was used to convert optical density data into hemoglobin signals in an arbitrary unit. Raw data were bandpass filtered between $0.04 \mathrm{~Hz}$ and $0.3 \mathrm{~Hz}$ to remove baseline drift and physiological noise (e.g., HR). Since oxy-Hb signals have a better signal-to-noise ratio than deoxy- $\mathrm{Hb}$, and deoxy- $\mathrm{Hb}$ signals have not shown significant Stroop interference effects in some fNIRS studies [42,43], only the oxy-Hb signals were used to monitor regional cortex activities [44]. The mean values of the oxy- $\mathrm{Hb}$ signals of the baseline $(0-2 \mathrm{~s}$ before the onset of the trial) and the task (Stroop task: 4-13 s after the onset of the trial; task-switching: 4-12 s after the onset of the trial) were computed for each subject, channel, and task condition. In addition, individual hemodynamic responses were adjusted to the mean value of the baseline.

\subsection{Anthropometrics and Physical Activity}

The participants' body weight and height were measured using standard protocols. Body composition was measured using an Inbody 720 Body Composition Analyzer (InBody Bldg., Seoul, Korea). The participants' daily physical activity level was surveyed using the short form of an International Physical Activity Questionnaire (IPAQ-SF) [45]. The metabolic equivalent (MET) scores were calculated by standardized procedures. 


\subsection{Statistics}

The statistical analyses were performed using IBM SPSS software for Windows. Assumptions of data normality were examined graphically. The potential differences in the participants' characteristics were tested by an unpaired sample t-test. The performances on the behavioral outcomes (i.e., accuracy and RT) and the fNIRS data were first assessed by a repeated-measures analysis of variance (RM-ANOVA). The paired sample t-test was conducted to assess the potential interaction effects between experiment and task conditions. For the Stroop interference effects and the switch costs (global and local), a paired sample t-Test was conducted between the experimental conditions (sitting/cycling). A false discovery rate (FDR) based on multiple testing adjustments was applied to the fNIRS data [46]. The statistical significance levels for all comparisons were set at $P<0.05$ (two-sided).

\section{Results}

\subsection{Participants Characteristics and Physiological Parameters}

The participants' characteristics were summarized by gender, shown in Table 1 . The males were taller than the females, and the females had greater body fat percentage (all $P<0.05)$.

Table 1. Participants' characteristics.

\begin{tabular}{ccccc}
\hline Characteristics & Men $(\boldsymbol{n}=\mathbf{2 0})$ & $\begin{array}{c}\text { Women }(\boldsymbol{n}= \\
\mathbf{1 7})\end{array}$ & All $(\boldsymbol{n = 3 7 )}$ & $\begin{array}{c}\boldsymbol{P} \text { for } \\
\text { gender }\end{array}$ \\
\hline Age (years) & $21.0 \pm 2.9$ & $22.4 \pm 1.6$ & $21.6 \pm 2.5$ & 0.08 \\
Height (cm) & $174.4 \pm 6.0$ & $166.4 \pm 5.3$ & $170.7 \pm 6.9$ & $<0.001$ \\
Weight (kg) & $64.0 \pm 14.8$ & $58.5 \pm 8.1$ & $61.4 \pm 12.3$ & 0.18 \\
BMI (kg/m2) & $22.1 \pm 2.1$ & $21.5 \pm 3.1$ & $21.8 \pm 2.6$ & 0.46 \\
Rest heart rate (BPM) & $76.2 \pm 11.3$ & $75.8 \pm 10.3$ & $76.0 \pm 10.7$ & 0.93 \\
Body fat percentage (\%) & $17.6 \pm 5.4$ & $23.1 \pm 5.8$ & $20.2 \pm 6.2$ & 0.003 \\
Physical activity & $3021(400-15,600)$ & $2506(480-5432)$ & $2785(400-15,600)$ & 0.57 \\
(MET-min/week) & & & & \\
\hline
\end{tabular}

Note: BMI, body mass index; BPM, beats per minute; MET, metabolic equivalent. Data are expressed as mean \pm standard deviation (SD) or median (interquartile range).

The cycling condition resulted in an increased HR (Stroop task: $81.1 \pm 1.8 \mathrm{BPM}$ vs. $73.3 \pm 1.8$ BPM, $P=0.002$; task-switching: $83.8 \pm 1.8 \mathrm{BPM}$ vs. $75.1 \pm 1.3 \mathrm{BPM}, P<0.001)$ compared with the sitting condition. However, the RPE scores were not significantly different between the cycling and the sitting condition at pre-test $(10.0 \pm 2.7$ vs. $10.0 \pm 2.8, P=0.80)$ and post-test $(12.5 \pm 2.5$ vs. 12.5 vs. 2.2 , $P=0.76)$.

\subsection{Behavioral Results}

\subsubsection{Stroop Task}

A RM-ANOVA performed on the accuracy of the Stroop task revealed that there were no significant main interaction effects. For RT, the RM-ANOVA revealed the main effects of the task condition $(\mathrm{F}(3,108)=37.25, P<0.05)$, indicating that the RT of the neutral task was significantly smaller than that of the incongruent task (Figure 3). These results indicated that the Stroop interference effects were observed between the neutral task and the incongruent task. Post hoc analyses revealed that, in the sitting condition, the neutral task did not have greater accuracy than the incongruent task $(94.31 \pm 5.84 \%$ vs. $93.19 \pm 7.13 \%, P=0.32)$. However, the RT of the neutral task was significantly smaller than that of the incongruent task $(670.55 \pm 214.80 \mathrm{~ms}$ vs. $800.48 \pm 274.80 \mathrm{~ms}, P<0.001)$. In the cycling condition, the accuracy was not significantly different between the neutral task and the incongruent task $(95.00 \pm 5.83 \%$ vs. $93.19 \pm 8.94 \%, P=0.07)$. The RT of the neutral task was 
significantly smaller than that of the incongruent task $(652.05 \pm 203.52 \mathrm{~ms}$ vs. $766.76 \pm 280.40 \mathrm{~ms}$, $P<0.001)$.
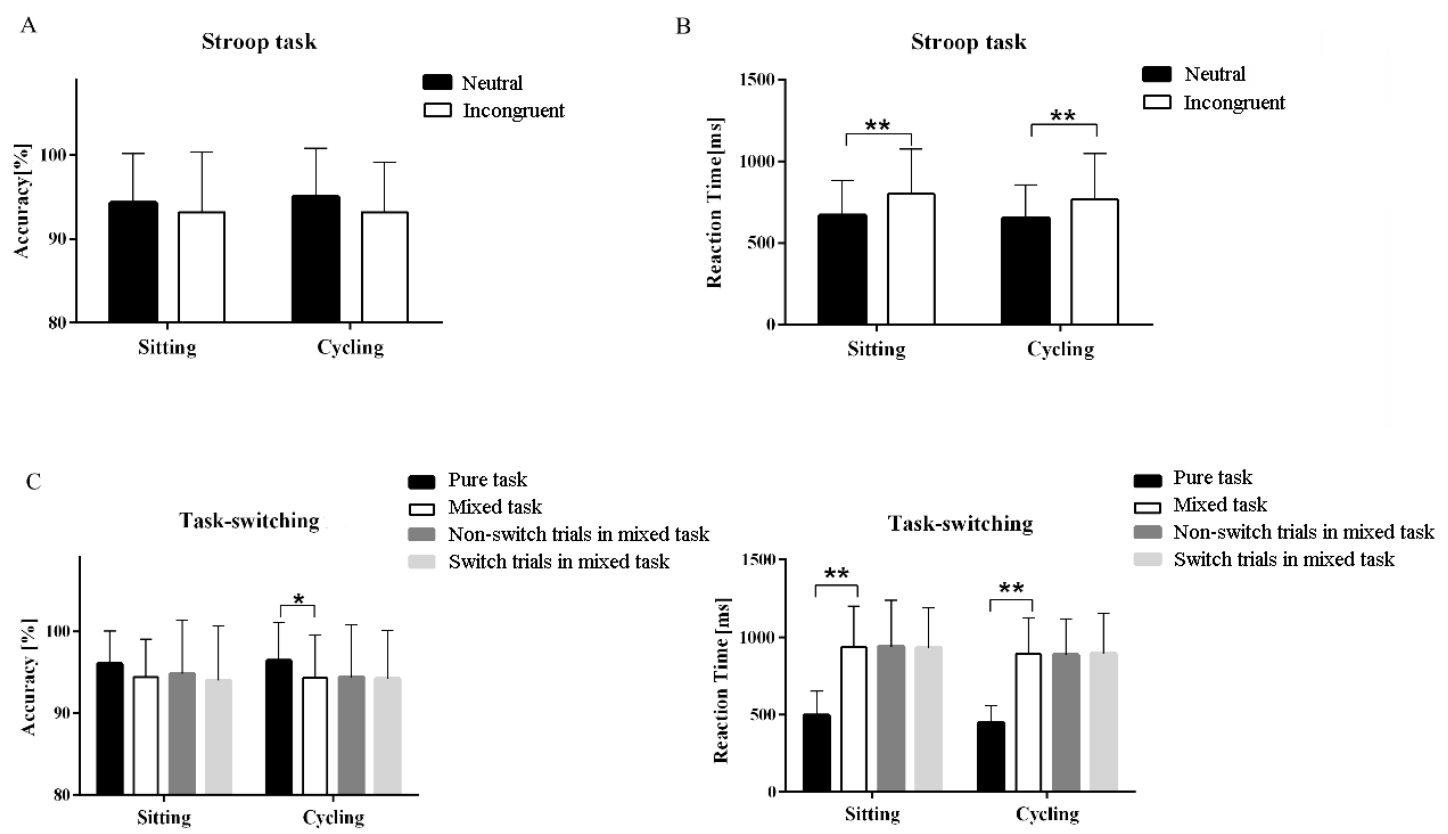

Figure 3. Behavioral results of the Stroop task and the task-switching (A) accuracy of Stroop task, (B) reaction time of Stroop task, (C) accuracy of task-switching, and (D) reaction time of task-switching. Data are expressed as mean $\pm \mathrm{SD}$. * indicates $P<0.05 ;{ }^{* *}$ indicates $P<0.01$ ).

The Stroop interference effects were derived and used to examine the potential differences between the experimental conditions. There were no significant differences in the Stroop interference effects between the sitting condition and the cycling condition (114.70 $\pm 141.99 \mathrm{~ms}$ vs. $129.93 \pm 124.10 \mathrm{~ms}$, $P=0.66)$.

\subsubsection{Task-Switching}

As shown in Figure 3, the RM-ANOVA performed on the accuracy of task-switching revealed that there were no significant main interaction effects. For RT, the RM-ANOVA demonstrated significant main effects of the task condition $(\mathrm{F}(3,108)=172.82, P<0.05)$, indicating that the RT of the pure task was significantly smaller than that of mixed task. Post-hoc analyses demonstrated that, in the sitting condition, the accuracy of the pure task was not significantly higher than that of the mixed task. The RT of the pure task was significantly smaller than that of the mixed task $(497.27 \pm 154.09 \mathrm{~ms}$ vs. $936.24 \pm 264.12 \mathrm{~ms}, P<0.001)$. In the cycling condition, the accuracy of the pure task was significantly higher than that of the mixed task $(96.36 \pm 4.67 \%$ vs. $94.27 \pm 5.26 \%, P=0.03)$. The RT of the pure task was significantly smaller than that the mixed task $(446.25 \pm 111.56 \mathrm{~ms}$ vs. $891.22 \pm 232.56 \mathrm{~ms}$, $P<0.001$ ).

There were no significant differences in the local switch costs $(-8.20 \pm 175.71 \mathrm{~ms}$ vs. $9.73 \pm 155.90 \mathrm{~ms}, P=0.67)$ and the global switch costs $(438.98 \pm 203.61 \mathrm{~ms}$ vs. $444.97 \pm 174.43 \mathrm{~ms}, P=$ $0.90)$ between the sitting and the cycling conditions.

\section{3. fNIRS Results}

\subsubsection{Stroop Task}

The RM-ANOVA performed on channel-wise oxy- $\mathrm{Hb}$ concentrations revealed significant main effects of the experimental condition in channel $5(\mathrm{~F}(1,36)=5.24, P<0.05$, FDR-corrected). The RM-ANOVA also revealed significant main interaction effects of the experimental conditions 
(sitting/cycling) $\times$ task condition in channel $5(\mathrm{~F}(1,36)=4.60, P<0.05$, FDR-corrected); channel 10 $(\mathrm{F}(1,36)=5.50, P<0.05$, FDR-corrected); and channel $12(\mathrm{~F}(1,36)=5.29, P<0.05$, FDR-corrected $)$. Post hoc analyses demonstrated that the oxy-Hb concentrations in response to the incongruent task in channel 10 were significantly higher than the neutral task (channel 10: $0.76 \pm 0.93$ vs. $1.22 \pm 1.29$, $P=0.02$, FDR-corrected) in the sitting condition. In the cycling condition, there were no significant differences in oxy- $\mathrm{Hb}$ responses between the neutral task and the incongruent task.

In channel 5, 10, and 12 (Figure 4), the cycling condition resulted in lower oxy-Hb associated with the Stroop interference effects (oxy- $\mathrm{Hb}$ interference) than that of the sitting condition channel 5 $(0.52 \pm 1.42$ vs. $-0.29 \pm 1.16, P<0.05$, FDR-corrected); channel 10 ( $0.54 \pm 1.03$ vs. $0.01 \pm 0.80, P<0.05$, FDR-corrected); and channel 12 ( $0.42 \pm 0.91$ vs. $-0.13 \pm 0.72, P<0.05$, FDR-corrected). The three channels were located approximately at the frontopolar area and the left-dorsolateral prefrontal cortex (L-DLPFC).
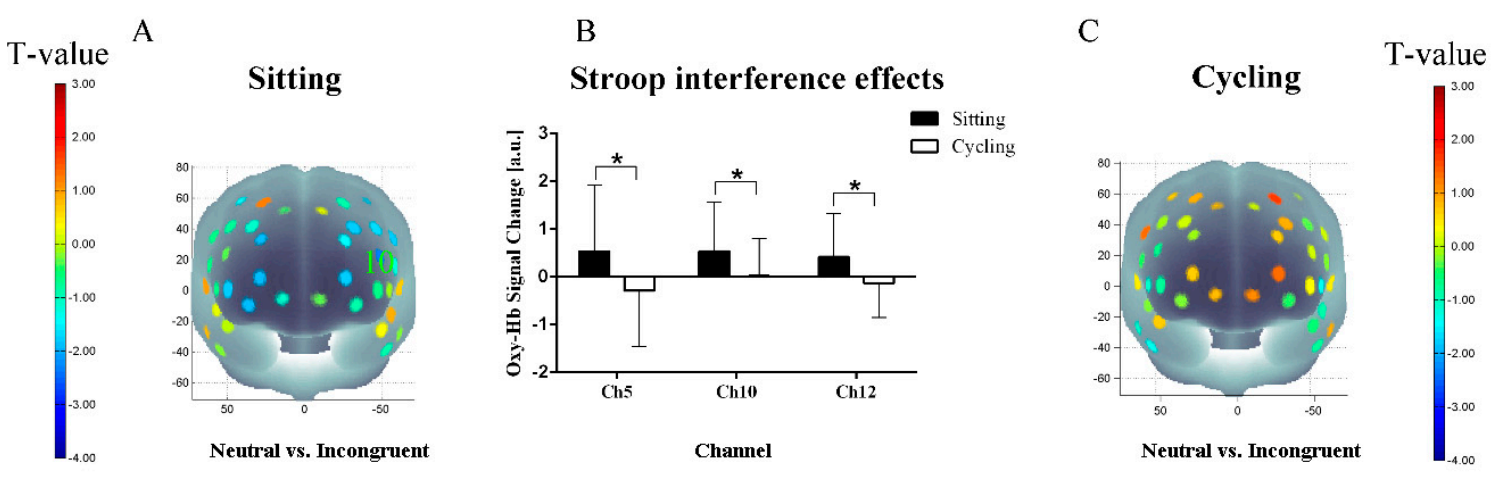

Figure 4. fNIRS results of the Stroop task (A) t-map of oxygenated hemoglobin (oxy-Hb) concentrations in the sitting condition; (B) Oxy-Hb changes related to the Stroop interference effects; (C) t-map of oxy- $\mathrm{Hb}$ concentrations in the cycling condition. Data are expressed as mean $\pm \mathrm{SD}$. * indicates FDR-corrected $P<0.05)$.

\subsubsection{Task-Switching}

The RM-ANOVA performed on channel-wise oxy-Hb concentrations revealed significant main effects of the task condition in the following: channel $3(\mathrm{~F}(3,108)=5.67, P<0.05$, FDR-corrected); channel $4(\mathrm{~F}(3,108)=3.26, P<0.05$, FDR-corrected); channel $10(\mathrm{~F}(3,108)=6.66, P<0.05$, FDR-corrected); channel 15 (F $(3,108)=4.60, P<0.05$, FDR-corrected); channel $16(\mathrm{~F}(3,108)=$ 4.74, $P<0.05$, FDR-corrected); channel $17(\mathrm{~F}(3,108)=5.80, P<0.05$, FDR-corrected); channel 24 (F $(3,108)=4.76, P<0.05$, FDR-corrected $)$; and channel $31(\mathrm{~F}(3,108)=5.68, P<0.05$, FDR-corrected $)$. A significant experiment condition (sitting/cycling) $\times$ task condition (pure/mixed) interaction was also observed in channel $3(\mathrm{~F}(3,108)=2.89, P<0.05$, FDR-corrected); channel $18(\mathrm{~F}(3,108)=4.94$, $P<0.01$, FDR-corrected); channel 29 (F $(3,108)=6.14, P<0.05$, FDR-corrected); and channel 31(F $(3$, 108) $=5.95, P<0.05$, FDR-corrected).

As shown in Figure 5, post hoc analyses demonstrated that the oxy-Hb concentrations in response to the mixed task and the pure task significantly differed in channels 16, 17, and 24 in the sitting condition (all $P<0.05$, FDR-corrected). In the cycling condition, the oxy-Hb concentrations in response to the mixed task were significantly higher than the pure task in channels $3,4,10,15,16,24,25,29$, and 31 (all $\mathrm{P}<0.05$, FDR-corrected). Meanwhile, in the cycling condition, the oxy-Hb concentrations in response to the switch trials in channels 4 and 18, were higher than those in response to the non-switch trials (all $P<0.05$, FDR-corrected). 

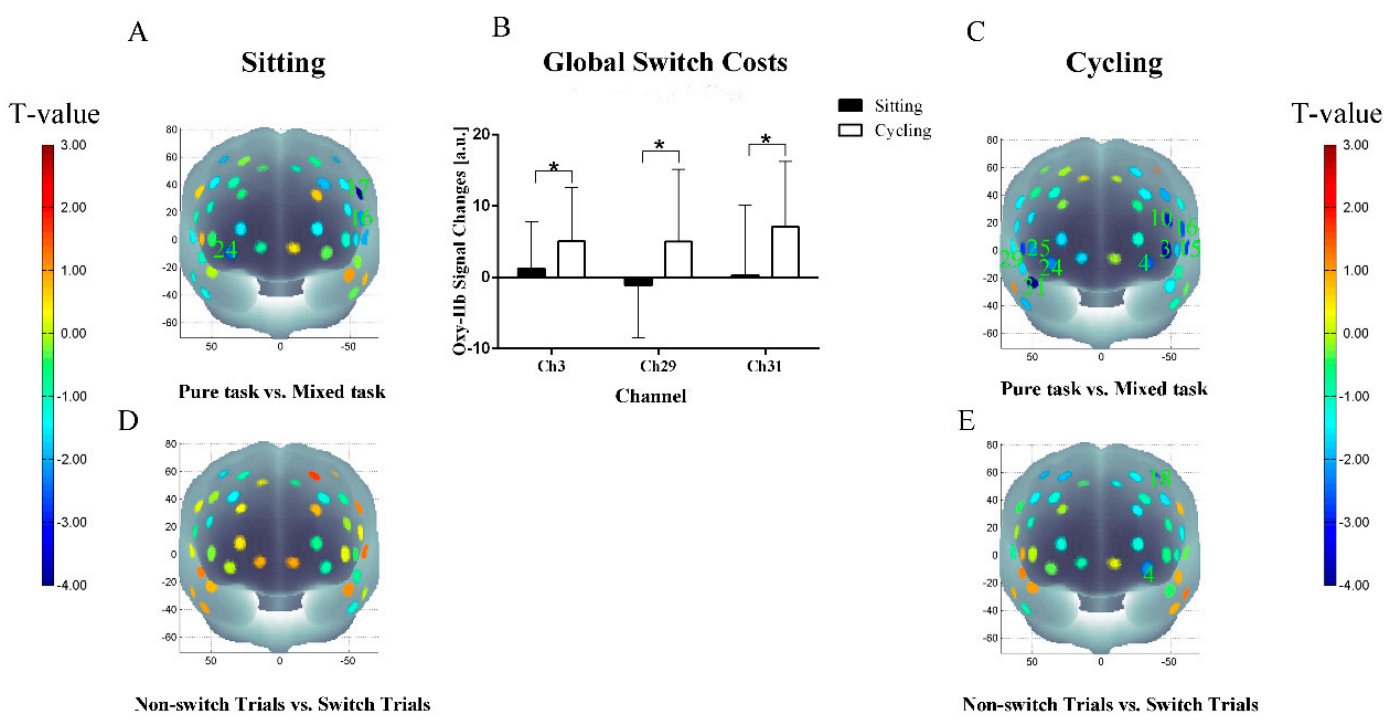

Figure 5. fNIRS results of task-switching (A) t-map of oxy-Hb concentrations in the sitting condition, pure task vs. mixed task; (B) Oxy-Hb changes related to the global switch costs; (C) t-map of oxy-Hb concentrations in the cycling condition, pure task vs. mixed task; (D) t-map of oxy-Hb concentrations in the sitting condition, non-switch trials vs. switch trials; (E) t-map of oxy-Hb concentrations in the cycling condition, non-switch trials vs. switch trials. Data are expressed as mean $\pm \mathrm{SD}$. * indicates FDR-corrected $P<0.05$.

As shown in Figure 5B, in channel 3 (approximately located in frontopolar area), channel 29 (approximately located in right ventrolateral prefrontal cortex, VLPFC), and channel 31 (approximately located in temporopolar area), the cycling condition resulted in a higher oxy-Hb concentration (change) associated with the global switch costs than with the sitting condition in channel $3(1.26 \pm 6.55$ vs. $5.05 \pm 7.48, P<0.05$, FDR-corrected); channel 29 ( $-1.11 \pm 7.40$ vs. $5.05 \pm 10.11, P<0.05$, FDR-corrected); and channel 31 ( $0.33 \pm 9.79$ vs. $7.11 \pm 9.14, P<0.05$, FDR-corrected). There were no significant differences in the local switch costs related to the oxy- $\mathrm{Hb}$ concentrations between the sitting and the cycling conditions.

\section{Discussion}

The present study aimed to determine whether self-paced cycling at an active workstation influences concurrent performance on executive functions and cortical activity in young adults. The study results demonstrated that the behavioral performances on the Stroop task and the task-switching paradigm were not influenced by cycling at an active workstation. The cortical activity pattern was changed when conducting two cognitive tasks while cycling at an active workstation.

In the current study, the RT, the accuracy of the Stroop task, and the Stroop interference effects did not differ between the two experimental conditions. For the task-switching, the RT and the accuracy of the pure task and the mixed task, as well as the global switch costs and the local switch costs were not different between either of the two experimental conditions. The results indicated that self-paced exercise at an active workstation did not impair the concurrent performances on executive functions. It was hypothesized that there is an inverted-U relationship between exercise-induced arousal and cognitive performance [47]. In this study, self-paced cycling was performed at a low-intensity without an obvious increase in HR and RPE, which indicates that self-paced cycling did not lead to a significant increase in arousal. Therefore, the unchanged arousal levels may partly explain the reasons for the behavioral results. Although the cognitive measures are not the same, the behavioral results of the present study are in line with several previous studies. A study of young adults showed that slow walking $(1.5 \mathrm{mph})$ on a treadmill desk did not affect concurrent performance on executive functions measured by the Eriksen flanker task and the go/no-go task [29]. Similarly, Ehmann et al. [28] found 
that, in young adults, self-selected low-intensity walking on an active workstation did not affect performance on the tests of inhibitory control, working memory, cognitive flexibility, and global executive functions. Pilcher et al. [30] reported that, in young college students, there were no differences on meta-cognitive measures between using an activity workstation with low-intensity cycling and using a traditional desk. It is also worth noting that some studies observed that the cognitive performance was influenced when using an active workstation. Interestingly, Torbeyns et al. [31] showed that, compared with a sitting condition, cycling at 30\% maximal external power improved the performance of adults on the Stroop task. In contrast, Zhang et al. [32] investigated the effects of standing and walking on self-paced speed $(2.3 \mathrm{~km} / \mathrm{h})$ and faster speed $(3.5 \mathrm{~km} / \mathrm{h})$ on inhibitory control, working memory, and cognitive flexibility in young adults. Their study showed that walking on a workstation with self-paced speed or faster speed selectively impaired performance of working memory measured by the N-back task, with no influence on inhibitory control and cognitive flexibility.

Although the behavioral performances did not differ between the sitting and the cycling conditions in the current study, it is important to clarify whether the in-task neural processing was affected when cycling using an active workstation. The fNIRS results showed that oxy-Hb concentrations associated with the Stroop interference effects and the global switch costs were different in some brain areas between the sitting and the cycling conditions. The results indicated that the dual-task condition (cycling condition) led to changes in cortical neural activity in relation to the Stroop task and the task-switching. Therefore, the findings suggest that the cognitive resources were re-allocated during the cycling condition. It is interesting that the pattern of changes in cortical activity differed between the Stroop task and the task-switching paradigm. The oxy-Hb concentrations associated with the Stroop interference in the three channels were lower during the cycling condition, which indicated a competition of cognitive resources when performing the Stroop task while cycling at an active workstation. The findings may be explained by the limited-capacity information processing model $[48,49]$, which suggest that the attentional resource was re-allocated when performing two tasks simultaneously (in this study, a cognitive task and a motor task of cycling). In contrast to the Stroop task, the oxy-Hb concentrations associated with the global switch costs in three channels were higher during the cycling condition. This may be due to the fact that the event-related task-switching paradigm is not difficult for the young participants. People did not need much cognitive resource when performing the single cognitive task. However, performing task-switching while cycling leads to additional cognitive resource. Therefore, an increased cortical activity was observed during the cycling condition. It is not possible to directly compare our fNIRS results with others, since, to the best of our knowledge, no other studies examined the pattern of executive function-related cortical activation using fNIRS under the condition of cycling at an active workstation. However, a study [50], using EEG, investigated the effects of using an active treadmill desk (self-determined low-intensity speeds) on brain activation during an attention task. No significant differences of steady state visually evoked potentials (SSVEPs) were observed among the sitting, standing, and walking conditions. Obviously, more studies employing similar study designs are warranted to confirm the effects of using active workstations on brain activity and allocation patterns of cognitive resource.

Given the fact that there is an increasing prevalence of sedentary lifestyle, the findings of this study have practical implications. Specifically, the implementation of active workstations in working places may not sacrifice or interfere with executive functioning, whereas it increases daily energy expenditure and breaks prolonged sedentary time. However, considering the fNIRS results of changed cortical activity patterns, this study also indicated that the cognitive resource was reallocated when performing cognitive tasks while self-paced cycling. Therefore, future studies employing various exercise intensities are warranted to elucidate the upper limit of exercise intensity at which the cognitive performance is not influenced by using an active workstation. Furthermore, future studies should investigate the influences of using active workstation on cognitive performances and neural activity patterns in various age groups. 
The primary strength of the study is that the pattern of cortical activation while performing cognitive tasks was measured. It allowed the present study to not only examine the effects of using an active workstation on cognitive task performances but also the in-task neural processing. However, the findings of the study should be interpreted within the context of some limitations. First, the study was conducted under a simulated working environment rather than actual office workplace. Therefore, the generalization of the findings on executive function performance to actual work efficiency may be limited. However, it is well documented that executive functions are important for success in many aspects of life, including academic performance and productivity at work [23]. Secondly, the study only examined the acute effects of using an active workstation for a short period of time. Therefore, future studies are needed to clarify the long-term effects of the implementation of active workstations.

\section{Conclusions}

The study indicated that the behavioral performances on executive functions were not affected by cycling at an active workstation. However, the in-task cortical neural activity was changed in some brain areas, which indicated that cognitive resources were reallocated during cycling at an active workstation.

Author Contributions: Conceptualization, K.W. and T.H.; methodology, T.H., Z.D., Q.G., Y.X., and L.Z.; formal analysis, Q.G., Z.D., and T.H.; investigation, K.W., Z.C., L.Z., and T.H.; data curation, T.H., Q.G., Z.D., C.T., Y.X., J.Z., Z.C., and K.W.; writing-original draft preparation, T.H. and K.W.; writing-review and editing, T.H., Q.G., Z.D., C.T., Y.X., J.Z., L.Z., Z.C., and K.W.; visualization, D.Z. and Q.G.; supervision, K.W. and Z.C.; project administration, K.W. and Z.C.; funding acquisition, T.H.

Funding: This research was funded by the General Administration of Sport of China, grant number 2017B044.

Acknowledgments: The authors would like to thank the participants for their participation in the study.

Conflicts of Interest: The authors declare no conflict of interest.

\section{References}

1. Tremblay, M.S.; Aubert, S.; Barnes, J.D.; Saunders, T.J.; Carson, V.; Latimer-Cheung, A.E.; Chastin, S.F.M.; Altenburg, T.M.; Chinapaw, M.J.M. Sedentary Behavior Research Network (SBRN)-Terminology Consensus Project process and outcome. Int. J. Behav. Nutr. Phys. Act. 2017, 14, 75. [CrossRef] [PubMed]

2. de Rezende, L.F.; Rey-Lopez, J.P.; Matsudo, V.K.; do Carmo Luiz, O. Sedentary behavior and health outcomes among older adults: A systematic review. BMC Public Health 2014, 14, 333. [CrossRef] [PubMed]

3. Ding, D.; Lawson, K.D.; Kolbe-Alexander, T.L.; Finkelstein, E.A.; Katzmarzyk, P.T.; van Mechelen, W.; Pratt, M. The economic burden of physical inactivity: A global analysis of major non-communicable diseases. Lancet 2016, 388, 1311-1324. [CrossRef]

4. Shields, M.; Tremblay, M.S. Sedentary behaviour and obesity. Health Rep. 2008, 19, 19-30. [PubMed]

5. Fritschi, C.; Park, H.; Richardson, A.; Park, C.; Collins, E.G.; Mermelstein, R.; Riesche, L.; Quinn, L. Association Between Daily Time Spent in Sedentary Behavior and Duration of Hyperglycemia in Type 2 Diabetes. Biol. Res. Nurs. 2016, 18, 160-166. [CrossRef] [PubMed]

6. Dempsey, P.C.; Owen, N.; Biddle, S.J.H.; Dunstan, D.W. Managing Sedentary Behavior to Reduce the Risk of Diabetes and Cardiovascular Disease. Curr. Diabetes Rep. 2014, 14, 522. [CrossRef]

7. Hoare, E.; Milton, K.; Foster, C.; Allender, S. The associations between sedentary behaviour and mental health among adolescents: A systematic review. Int. J. Behav. Nutr. Phys. Act. 2016, 13, 108. [CrossRef] [PubMed]

8. Vancampfort, D.; Stubbs, B.; Lara, E.; Vandenbulcke, M.; Swinnen, N.; Smith, L.; Firth, J.; Herring, M.P.; Hallgren, M.; Koyanagi, A. Mild cognitive impairment and sedentary behavior: A multinational study. Exp. Gerontol. 2018, 108, 174-180. [CrossRef]

9. Benatti, F.B.; Ried-Larsen, M. The Effects of Breaking up Prolonged Sitting Time: A Review of Experimental Studies. Med. Sci. Sports Exerc. 2015, 47, 2053-2061. [CrossRef]

10. Hawari, N.S.A.; Wilson, J.; Gill, J.M.R. Effects of breaking up sedentary time with "chair squats" on postprandial metabolism. J. Sports Sci. 2018, 37, 331-338. [CrossRef] 
11. Torbeyns, T.; Bailey, S.; Bos, I.; Meeusen, R. Active workstations to fight sedentary behaviour. Sports Med 2014, 44, 1261-1273. [CrossRef] [PubMed]

12. Koren, K.; Pisot, R.; Simunic, B. Active workstation allows office workers to work efficiently while sitting and exercising moderately. Appl. Ergon. 2016, 54, 83-89. [CrossRef] [PubMed]

13. Johnston, V.; Gane, E.M.; Brown, W.; Vicenzino, B.; Healy, G.N.; Gilson, N.; Smith, M.D. Feasibility and impact of sit-stand workstations with and without exercise in office workers at risk of low back pain: A pilot comparative effectiveness trial. Appl. Ergon. 2019, 76, 82-89. [CrossRef] [PubMed]

14. Bastien Tardif, C.; Cantin, M.; Senecal, S.; Leger, P.M.; Labonte-Lemoyne, E.; Begon, M.; Mathieu, M.E. Implementation of Active Workstations in University Libraries-A Comparison of Portable Pedal Exercise Machines and Standing Desks. Int. J. Environ. Res. Public Health 2018, 15, 1242. [CrossRef]

15. Neuhaus, M.; Eakin, E.G.; Straker, L.; Owen, N.; Dunstan, D.W.; Reid, N.; Healy, G.N. Reducing occupational sedentary time: A systematic review and meta-analysis of evidence on activity-permissive workstations. Obes. Rev. 2015, 15, 822-838. [CrossRef]

16. Martin, A.; Fitzsimons, C.; Jepson, R.; Saunders, D.H.; Ploeg, H.P.V.D.; Teixeira, P.J.; Gray, C.M.; Mutrie, N. Interventions with potential to reduce sedentary time in adults: Systematic review and meta-analysis. $\mathrm{Br}$. J. Sports Med. 2015, 49, 1056. [CrossRef]

17. Koepp, G.A.; Manohar, C.U.; Mccradyspitzer, S.K.; Benner, A.; Hamann, D.J.; Runge, C.F.; Levine, J.A. Treadmill desks: A 1-year prospective trial. Obesity 2013, 21, 705-711. [CrossRef] [PubMed]

18. Torbeyns, T.; De, G.B.; Bailey, S.; De, P.K.; Decroix, L.; Van, C.J.; Meeusen, R. Bike Desks in the Office: Physical Health, Cognitive Function, Work Engagement, and Work Performance. J. Occup. Environ. Med. 2016, 58, 1257. [CrossRef]

19. John, D.; Thompson, D.L.; Raynor, H.; Bielak, K.; Rider, B.; Bassett, D.R. Treadmill workstations: A worksite physical activity intervention in overweight and obese office workers. J. Phys. Act. Health 2011, 8, 1034-1043. [CrossRef]

20. Miyashita, M.; Park, J.H.; Takahashi, M.; Suzuki, K.; Stensel, D.; Nakamura, Y. Postprandial lipaemia: Effects of sitting, standing and walking in healthy normolipidaemic humans. Int. J. Sports Med. 2013, 34, $21-27$. [CrossRef]

21. Dunstan, D.W.; Kingwell, B.A.; Larsen, R.; Healy, G.N.; Cerin, E.; Hamilton, M.T.; Shaw, J.E.; Bertovic, D.A.; Zimmet, P.Z.; Salmon, J. Breaking Up Prolonged Sitting Reduces Postprandial Glucose and Insulin Responses. Diabetes Care 2012, 35, 976-983. [CrossRef] [PubMed]

22. Ojo, S.O.; Bailey, D.P.; Chater, A.M.; Hewson, D.J. The Impact of Active Workstations on Workplace Productivity and Performance: A Systematic Review. Int. J. Environ. Res. Public Health 2018, 15, 417. [CrossRef] [PubMed]

23. Diamond, A. Executive functions. Annu. Rev. Psychol. 2013, 64, 135-168. [CrossRef] [PubMed]

24. Riggs, N.R.; Jahromi, L.B.; Razza, R.P.; Dillworth-Bart, J.E.; Mueller, U. Executive function and the promotion of social-emotional competence. J. Appl. Dev. Psychol. 2006, 27, 300-309. [CrossRef]

25. Wiebe, S.A.; Karbach, J. Executive Function: Development Across the Life Span; Routledge: Abingdon, UK, 2017.

26. Alderman, B.L.; Olson, R.L.; Mattina, D.M. Cognitive function during low-intensity walking: A test of the treadmill workstation. J. Phys. Act. Health 2014, 11, 752. [CrossRef] [PubMed]

27. Ohlinger, C.M.; Horn, T.S.; Berg, W.P.; Cox, R.H. The effect of active workstation use on measures of cognition, attention, and motor skill. J. Phys. Act. Health 2011, 8, 119-125. [CrossRef]

28. Ehmann, P.J.; Brush, C.J.; Olson, R.L.; Bhatt, S.N.; Banu, A.H.; Alderman, B.L. Active Workstations Do Not Impair Executive Function in Young and Middle-Age Adults. Med. Sci. Sports Exerc. 2017, 49, 965-974. [CrossRef] [PubMed]

29. Larson, M.J.; LeCheminant, J.D.; Carbine, K.; Hill, K.R.; Christenson, E.; Masterson, T.; LeCheminant, R. Slow walking on a treadmill desk does not negatively affect executive abilities: An examination of cognitive control, conflict adaptation, response inhibition, and post-error slowing. Front. Psychol. 2015, 6, 723. [CrossRef] [PubMed]

30. Pilcher, J.J.; Baker, V.C. Task Performance and Meta-Cognitive Outcomes When Using Activity Workstations and Traditional Desks. Front. Psychol. 2016, 7, 957. [CrossRef]

31. Torbeyns, T.; de Geus, B.; Bailey, S.; De Pauw, K.; Decroix, L.; Van Cutsem, J.; Meeusen, R. Cycling on a Bike Desk Positively Influences Cognitive Performance. PLoS ONE 2016, 11, e0165510. [CrossRef] [PubMed] 
32. Zhang, Z.; Zhang, B.; Cao, C.; Chen, W. The effects of using an active workstation on executive function in Chinese college students. PLoS ONE 2018, 13, e0197740. [CrossRef] [PubMed]

33. Tempest, G.D.; Reiss, A.L. The Utility of fNIRS for Measuring Cortical Activity during Cycling-Exercise. Med. Sci. Sports Exerc. 2018. [CrossRef] [PubMed]

34. Sun, J.; Sun, B.; Zhang, L.; Luo, Q.; Gong, H. Correlation between hemodynamic and electrophysiological signals dissociates neural correlates of conflict detection and resolution in a Stroop task: A simultaneous near-infrared spectroscopy and event-related potential study. J. Biomed. Opt. 2013, 18, 096014. [CrossRef]

35. Kujach, S.; Byun, K.; Hyodo, K.; Suwabe, K.; Fukuie, T.; Laskowski, R.; Dan, I.; Soya, H. A transferable high-intensity intermittent exercise improves executive performance in association with dorsolateral prefrontal activation in young adults. Neuroimage 2018, 169, 117-125. [CrossRef] [PubMed]

36. Cutini, S.; Scatturin, P.; Menon, E.; Bisiacchi, P.S.; Gamberini, L.; Zorzi, M.; Dell'Acqua, R. Selective activation of the superior frontal gyrus in task-switching: An event-related fNIRS study. Neuroimage 2008, 42, 945-955. [CrossRef]

37. Kray, J.; Lindenberger, U. Adult age differences in task switching. Psychol. Aging 2000, 15, 126-147. [CrossRef] [PubMed]

38. Themanson, J.R.; Hillman, C.H.; Curtin, J.J. Age and physical activity influences on action monitoring during task switching. Neurobiol. Aging 2006, 27, 1335-1345. [CrossRef]

39. Okamoto, M.; Dan, H.; Sakamoto, K.; Takeo, K.; Shimizu, K.; Kohno, S.; Oda, I.; Isobe, S.; Suzuki, T.; Kohyama, K.; et al. Three-dimensional probabilistic anatomical cranio-cerebral correlation via the international 10-20 system oriented for transcranial functional brain mapping. Neuroimage 2004, 21, 99-111. [CrossRef]

40. Tsuzuki, D.; Jurcak, V.; Singh, A.K.; Okamoto, M.; Watanabe, E.; Dan, I. Virtual spatial registration of stand-alone fNIRS data to MNI space. Neuroimage 2007, 34, 1506-1518. [CrossRef]

41. Huppert, T.J.; Diamond, S.G.; Franceschini, M.A.; Boas, D.A. HomER: A review of time-series analysis methods for near-infrared spectroscopy of the brain. Appl. Opt. 2009, 48, D280-D298. [CrossRef]

42. Schroeter, M.L.; Zysset, S.; Kupka, T.; Kruggel, F.; Yves von Cramon, D. Near-infrared spectroscopy can detect brain activity during a color-word matching Stroop task in an event-related design. Hum. Brain Mapp. 2002, 17, 61-71. [CrossRef]

43. Byun, K.; Hyodo, K.; Suwabe, K.; Ochi, G.; Sakairi, Y.; Kato, M.; Dan, I.; Soya, H. Positive effect of acute mild exercise on executive function via arousal-related prefrontal activations: An fNIRS study. Neuroimage 2014, 98, 336-345. [CrossRef]

44. Strangman, G.; Culver, J.P.; Thompson, J.H.; Boas, D.A. A quantitative comparison of simultaneous BOLD fMRI and NIRS recordings during functional brain activation. Neuroimage 2002, 17, 719-731. [CrossRef]

45. Fan, M.; Lyu, J.; He, P. Chinese guidelines for data processing and analysis concerning the International Physical Activity Questionnaire. Zhonghua Liu Xing Bing Xue Za Zhi 2014, 35, 961-964.

46. Glickman, M.E.; Rao, S.R.; Schultz, M.R. False discovery rate control is a recommended alternative to Bonferroni-type adjustments in health studies. J. Clin. Epidemiol. 2014, 67, 850-857. [CrossRef] [PubMed]

47. Lambourne, K.; Tomporowski, P. The effect of exercise-induced arousal on cognitive task performance: A meta-regression analysis. Brain Res. 2010, 1341, 12-24. [CrossRef] [PubMed]

48. Leone, C.; Feys, P.; Moumdjian, L.; D’Amico, E.; Zappia, M.; Patti, F. Cognitive-motor dual-task interference: A systematic review of neural correlates. Neurosci. Biobehav. Rev. 2017, 75, 348-360. [CrossRef] [PubMed]

49. Friedman, A.; Polson, M.C.; Dafoe, C.G.; Gaskill, S.J. Dividing attention within and between hemispheres: Testing a multiple resources approach to limited-capacity information processing. J. Exp. Psychol. Hum. Percept. Perform. 1982, 8, 625-650. [CrossRef] [PubMed]

50. Gilson, N.D.; Hall, C.; Renton, A.; Ng, N.; von Hippel, W. Do Sitting, Standing, or Treadmill Desks Impact Psychobiological Indicators of Work Productivity? J. Phys. Act. Health 2017, 14, 793-796. [CrossRef] [PubMed]

(C) 2019 by the authors. Licensee MDPI, Basel, Switzerland. This article is an open access article distributed under the terms and conditions of the Creative Commons Attribution (CC BY) license (http:// creativecommons.org/licenses/by/4.0/). 